\title{
De un Modelo Ondulatorio a un Modelo Difusivo: convergencia puntual de soluciones
}

\author{
From a Wave Model to a Diffusive Model: Point convergence of solutions \\ Juan Carlos Cordero Ceballos, Ricardo Pinilla Estupiñan \\ Departamento de Matemáticas y Estadística, Universidad Nacional de Colombia, Manizales, Colombia \\ Correo-e: jccorderoc@unal.edu.co, rpinillae@unal.edu.co
}

\begin{abstract}
Resumen-Consideramos el modelo lineal con coeficientes constantes para las vibraciones transversales de una cuerda elástica en ausencia de fuerzas externas, también asociado al problema de una línea de transmisión sin disipación. Probaremos que para cierto tipo de datos iniciales las soluciones de dicho modelo se aproximan puntualmente a la solución de la ecuación de onda o bien a la solución de la ecuación del calor, dependiendo de la elección del parámetro limite. Estos son los limites no viscoso y de densidad cero respectivamente. Como consecuencia, podemos decir que los modelos clásicos ondulatorio y difusivo se conectan vía la ecuación del telégrafo.
\end{abstract}

Palabras clave- Coeficiente de viscosidad, convergencia dominada, densidad lineal, ecuación característica, integral de Lebesgue.

\begin{abstract}
We consider the linear model with constant coefficients (parameters) for the transverse vibrations of an elastic string in absence of external forces, also related to the problem of a transmission line without dissipation. We will prove that, for some kind of initial data, the solutions of such model converge pointwise to the solution of the wave equation or to the solution of the heat equation, depending on the choice of the limit parameter. Those limits are the no-viscous one and the zero density one, respectively. As a consequence, we can say that the classical models, the wave one and the diffusive one, are connected by means of the telegrapher's equation.
\end{abstract}

Key Word - Coefficient of viscosity, dominated convergence, linear density, characteristic equation, Lebesgue integral

\section{INTRODUCCIÓN}

Las vibraciones transversales de una cuerda elástica unidimensional en ausencia de fuerzas externas pueden ser modeladas por la ecuación diferencial parcial lineal

$$
\alpha \partial_{t}^{2} u-\beta \partial_{x}^{2} u+\gamma \partial_{t} u=0
$$

donde $u=u(x, t)$ representa la amplitud de la cuerda en la posición $x \mathrm{y}$ al instante $t$. Los coeficientes $\alpha, \beta, \gamma \geq 0$ son parámetros que dependen de las propiedades del material que constituye la cuerda, pero no de $x$ ni de $t$.

Específicamente, $\alpha$ es la densidad (lineal) de masa, $\gamma$ es un coeficiente de viscosidad del medio y $\beta$ es una tensión inicial de la cuerda en equilibrio.

La ecuación (1) también es conocida como la ecuación del telégrafo, ver $[2,5]$.

Formalmente, si en (1) hacemos que $\alpha \rightarrow 0$ obtenemos

$$
\gamma \partial_{t} u-\beta \partial_{x}^{2} u=0
$$

la conocida ecuación del calor ó de difusión.

De otra forma, si en (1) hacemos que $\gamma \rightarrow 0$ obtenemos la ecuación

$$
\alpha \partial_{t}^{2} u-\beta \partial_{x}^{2} u=0
$$

llamada clásicamente ecuación de onda.

El objetivo del presente trabajo es probar que soluciones de la ecuación (1) convergen puntualmente para soluciones de la ecuación (2) cuando $\alpha \rightarrow 0$, ó bién para soluciones de la ecuación (3) si $\gamma \rightarrow 0$. Más exactamente, consideramos soluciones del PVI 
$\left\{\begin{array}{l}\alpha \partial_{t}^{2} u-\beta \partial_{x}^{2} u+\gamma \partial_{t} u=0 \text { en } \mathbb{R} \times(0,+\infty), \\ u=g_{\alpha \gamma}, \partial_{t} u=h_{\alpha \gamma} \quad \text { sobre } \mathbb{R} \times\{t=0\} .\end{array}\right.$ (4) $\left\{\begin{array}{c}\alpha \partial_{t}^{2} u-\beta \partial_{x}^{2} u+\gamma \partial_{t} u=0 \text { en } \mathbb{R} \times(0,+\infty), \\ u=g_{\alpha \gamma}, \partial_{t} u=h_{\alpha \gamma} \quad \text { sobre } \mathbb{R} \times\{t=0\},\end{array}\right.$

que denotaremos $u_{\alpha \gamma}$ y mostraremos que

$$
\begin{aligned}
& \lim _{\alpha \rightarrow 0} u_{\alpha \gamma}(x, t)=u_{\gamma}, \\
& \lim _{\gamma \rightarrow 0} u_{\alpha \gamma}(x, t)=u_{\alpha},
\end{aligned}
$$

donde $u_{\gamma}$ es solución del PVI

$$
\left\{\begin{array}{l}
\gamma \partial_{t} u-\beta \partial_{x}^{2} u=0 \text { en } \mathbb{R} \times(0,+\infty) \\
u=g_{\gamma} \quad \text { sobre } \mathbb{R} \times\{t=0\}
\end{array}\right.
$$

con $\lim _{\alpha \rightarrow 0} g_{\alpha \gamma}=g_{\gamma}, \mathrm{y}$ donde $u_{\alpha}$ es solución del PVI

$$
\left\{\begin{array}{l}
\alpha \partial_{t}^{2} u-\beta \partial_{x}^{2} u=0 \quad \text { en } \mathbb{R} \times(0,+\infty) \\
u=g_{\alpha}, \partial_{t} u=h_{\alpha} \quad \text { sobre } \mathbb{R} \times\{t=0\}
\end{array}\right.
$$

con $\lim _{\gamma \rightarrow 0} g_{\alpha \gamma}=g_{\alpha} \mathrm{y} \lim _{\gamma \rightarrow 0} h_{\alpha \gamma}=h_{\alpha}$.

Los datos iniciales $g_{\alpha \gamma}$ y $h_{\alpha \gamma}$ se escogen de tal manera que decrecen más rápido que un polinomio.

Hemos organizado nuestro trabajo de tal manera que en la sección 2 solucionamos los PVI's (4), (5) y (6) por medio de la transformada de Fourier y en la sección 3 presentamos nuestro resultado principal. Finalmente en la sección 4 hacemos una breve conclusión de lo que se hizo y proponemos futuras posibilidades que mejoren nuestro resultado.

\section{SOLUCIÓN POR TRANSFORMADA DE FOURIER}

En esta sección resolveremos los PVI's (4), (5) y (6) usando la transformada de Fourier.

Durante el desarrollo de este trabajo, $S(\mathbb{R})$ denota el espacio de Schwartz o de funciones rapidamente decrecientes, y $\hat{f}$ la transformada de Fourier en la variable espacial de la función $f$.

\section{A. SOLUCIÓN DE LA ECUACIÓN DEL TELÉGRAFO, PVI (4)}

Consideramos el PVI. donde $\alpha, \beta, \gamma \in(0, R], R \in \mathbb{R}^{+}$y $g_{\alpha \gamma}(x), h_{\alpha \gamma}(x)$, son tales que

$$
\hat{g}_{\alpha \gamma}(\xi)=\Phi_{\alpha \gamma}(\xi) \tilde{g}(\xi)
$$

$$
\hat{h}_{\alpha \gamma}=\Phi_{\alpha \gamma}(\xi) \tilde{h}(\xi)
$$

$\tilde{g}$ y $\tilde{h}$ son funciones en $S(\mathbb{R})$ dadas, y $\Phi_{\alpha \gamma}$ es un polinomio en la variable $\xi$.

Aplicando transformada de Fourier, obtenemos

$$
\begin{cases}\alpha \partial_{t}^{2} \hat{u}+\beta \xi^{2} \hat{u}+\gamma \partial_{t} \hat{u}=0 & \text { parat }>0 \\ \hat{u}=\widehat{g}_{\alpha \gamma}, \partial_{t} \hat{u}=\hat{h}_{\alpha \gamma} & \text { parat }=0\end{cases}
$$

Puesto que no intervienen derivadas con respecto a $\xi$, para cada $\xi$ fijo, (10) es de hecho un problema de valor inicial para una EDO de segundo orden, con coeficientes $\alpha, \beta \xi^{2}, \gamma$ constantes. Su ecuación característica es

$$
\alpha \lambda^{2}+\gamma \lambda+\beta \xi^{2}=0
$$

cuyas raíces son:

$$
\begin{aligned}
& \lambda_{1}=\frac{-\gamma+\sqrt{\gamma^{2}-4 \alpha \beta \xi^{2}}}{2 \alpha} \\
& \lambda_{2}=\frac{-\gamma-\sqrt{\gamma^{2}-4 \alpha \beta \xi^{2}}}{2 \alpha} .
\end{aligned}
$$

Vamos a suponer que la función $\Phi_{\alpha \gamma}$ que modula los datos iniciales (8) y (9) está dada por el discriminante de dicha ecuación, es decir, $\Phi_{\alpha \gamma}(\xi)=\gamma^{2}-4 \alpha \beta \xi^{2}$.

Dependiendo de si $\Phi_{\alpha \gamma}$ es mayor o menor que cero tenemos dos casos, el caso real y el caso complejo.

1. Caso real. Tenemos que $\Phi_{\alpha \gamma}(\xi) \geq 0$, es decir $\gamma^{2}-$ $4 \alpha \beta \xi^{2} \geq 0$, por tanto $|\xi| \leq \frac{\gamma}{2 \sqrt{\alpha \beta}}$, luego la solución de la EDO es:

$$
\hat{u}(\xi, t)=C_{1} \exp \left(\lambda_{1} t\right)+C_{2} \exp \left(\lambda_{2} t\right) .
$$


Considerando las condiciones iniciales tenemos el siguiente sistema

de donde

$$
\left\{\begin{array}{c}
\hat{g}_{\alpha \gamma}(\xi)=C_{1}+C_{2} \\
\hat{h}_{\alpha \gamma}(\xi)=\lambda_{1} C_{1}+\lambda_{2} C_{2}
\end{array}\right.
$$

$$
\begin{aligned}
& C_{1}=\alpha \sqrt{\gamma^{2}-4 \alpha \beta \xi^{2}} \tilde{h}(\xi)- \\
& \lambda_{2} \alpha \sqrt{\gamma^{2}-4 \alpha \beta \xi^{2}} \tilde{g}(\xi) \\
& C_{2}=-\left[\alpha \sqrt{\gamma^{2}-4 \alpha \beta \xi^{2}} \tilde{h}(\xi)-\right. \\
& \left.\lambda_{1} \alpha \sqrt{\gamma^{2}-4 \alpha \beta \xi^{2}} \tilde{g}(\xi)\right] .
\end{aligned}
$$

Reemplazando (14) y (15) en (13) tenemos

$$
\begin{aligned}
\hat{u}(\xi, t) & =\alpha \sqrt{\gamma^{2}-4 \alpha \beta \xi^{2}}(\tilde{h}(\xi) \\
- & \left.\lambda_{2} \tilde{g}(\xi)\right) \exp \left(\lambda_{1} t\right) \\
+ & \alpha \sqrt{\gamma^{2}-4 \alpha \beta \xi^{2}}\left(\lambda_{1} \tilde{g}(\xi)\right. \\
- & \tilde{h}(\xi)) \exp \left(\lambda_{1} t\right)
\end{aligned}
$$

y de (11) y (12) obtenemos

$$
\begin{aligned}
& \hat{u}(\xi, t)=\frac{\sqrt{\gamma^{2}-4 \alpha \beta \xi^{2}}}{2}\{[2 \alpha \tilde{h}(\xi)+ \\
& (\gamma+ \\
& \left.\left.\sqrt{\gamma^{2}-4 \alpha \beta \xi^{2}}\right) \tilde{g}(\xi)\right] \exp \left(\frac{-\gamma+\sqrt{\gamma^{2}-4 \alpha \beta \xi^{2}}}{2 \alpha} t\right)+(26) \\
& {\left[\left(-\gamma+\sqrt{\gamma^{2}-4 \alpha \beta \xi^{2}}\right) \tilde{g}(\xi)-\right.} \\
& \left.2 \alpha \tilde{h}(\xi)] \exp \left(\frac{-\gamma-\sqrt{\gamma^{2}-4 \alpha \beta \xi^{2}}}{2 \alpha} t\right)\right\}
\end{aligned}
$$

para $|\xi| \leq \frac{\gamma}{2 \sqrt{\alpha \beta}}$

2. Caso complejo. Tenemos que $\Phi_{\alpha \gamma}(\xi)<0$, es decir $\gamma^{2}-4 \alpha \beta \xi^{2}<0$, por tanto $|\xi|>\frac{\gamma}{2 \sqrt{\alpha \beta}} \mathrm{y}$

$$
\begin{aligned}
& \lambda_{1}=\frac{-\gamma+i \sqrt{4 \alpha \beta \xi^{2}-\gamma^{2}}}{2 \alpha}, \\
& \lambda_{2}=\frac{-\gamma-i \sqrt{4 \alpha \beta \xi^{2}-\gamma^{2}}}{2 \alpha} .
\end{aligned}
$$

En tal caso la solución de la EDO viene dada por

$$
\begin{aligned}
& \hat{\mathcal{V}}(\xi, t) \\
& =C_{1} \exp \left(\frac{-\gamma+i \sqrt{4 \alpha \beta \xi^{2}-\gamma^{2}}}{2 \alpha} t\right) \\
& +C_{2} \exp \left(\frac{-\gamma-i \sqrt{4 \alpha \beta \xi^{2}-\gamma^{2}}}{2 \alpha} t\right)
\end{aligned}
$$

para $|\xi|>\frac{\gamma}{2 \sqrt{\alpha \beta}}$

De las condiciones iniciales tenemos

$$
\begin{array}{r}
C_{1}=\frac{-i \sqrt{4 \alpha \beta \xi^{2}-\gamma^{2}}}{2}\{2 \alpha \tilde{h}(\xi) \\
+\tilde{g}(\xi)[-\gamma \\
\left.\left.-i \sqrt{4 \alpha \beta \xi^{2}-\gamma^{2}}\right]\right\} \\
C_{2}=\frac{-i \sqrt{4 \alpha \beta \xi^{2}-\gamma^{2}}}{2}\{2 \alpha \tilde{h}(\xi) \\
+\tilde{g}(\xi)[\gamma \\
\left.\left.-i \sqrt{4 \alpha \beta \xi^{2}-\gamma^{2}}\right]\right\} .
\end{array}
$$

Sustituyendo (18) y (19) en (17) obtenemos

$$
\begin{aligned}
& \hat{\mathcal{V}}(\xi, t)=\frac{-i \sqrt{4 \alpha \beta \xi^{2}-\gamma^{2}}}{2}\{\tilde{g}(\xi)[-\gamma- \\
& \left.i \sqrt{4 \alpha \beta \xi^{2}-\gamma^{2}}\right]- \\
& 2 \alpha \tilde{h}(\xi)\} \exp \left(\frac{-\gamma+i \sqrt{4 \alpha \beta \xi^{2}-\gamma^{2}}}{2 \alpha} t\right)- \\
& \frac{i \sqrt{4 \alpha \beta \xi^{2}-\gamma^{2}}}{2}\{2 \alpha \tilde{h}(\xi)+\tilde{g}(\xi)[\gamma- \\
& \left.\left.i \sqrt{4 \alpha \beta \xi^{2}-\gamma^{2}}\right]\right\} \exp \left(\frac{-\gamma-i \sqrt{4 \alpha \beta \xi^{2}-\gamma^{2}}}{2 \alpha} t\right)
\end{aligned}
$$

para $|\xi|>\frac{\gamma}{2 \sqrt{\alpha \beta}}$

Entonces podemos escribir

$\hat{u}(\xi, t)= \begin{cases}\hat{U}(\xi, t) & \text { si }|\xi| \leq \frac{\gamma}{2 \sqrt{\alpha \beta}}, \\ \hat{v}(\xi, t) & \text { si }|\xi|>\frac{\gamma}{2 \sqrt{\alpha \beta}},\end{cases}$

y aplicando transformada inversa de Fourier, obtenemos 


$$
\begin{aligned}
& u(x, t)=\frac{1}{\sqrt{2 \pi}} \int_{|\xi| \leq \frac{\gamma}{2 \sqrt{\alpha \beta}}} \hat{U}(\xi, t) \exp (x \xi i) d \xi+ \\
& \frac{1}{\sqrt{2 \pi}} \int_{|\xi|>\frac{\gamma}{2 \sqrt{\alpha \beta}} \hat{v}(\xi, t) \exp (x \xi i) d \xi .} \hat{x}+
\end{aligned}
$$

Definiendo las siguientes funciones característica

$$
\begin{gathered}
\chi_{\alpha \gamma}(\xi):= \begin{cases}1 & \text { si }|\xi| \leq \frac{\gamma}{2 \sqrt{\alpha \beta}} \\
0 & \text { si }|\xi|>\frac{\gamma}{2 \sqrt{\alpha \beta}}\end{cases} \\
\bar{\chi}_{\alpha \gamma}(\xi)= \begin{cases}1 & \text { si }|\xi|>\frac{\gamma}{2 \sqrt{\alpha \beta}} \\
0 & \text { si }|\xi| \leq \frac{\gamma}{2 \sqrt{\alpha \beta}},\end{cases}
\end{gathered}
$$

tenemos que

$$
\begin{aligned}
& u(x, t) \\
& =\frac{1}{\sqrt{2 \pi}} \int_{-\infty}^{+\infty} \chi_{\alpha \gamma}(\xi) \hat{\mathcal{u}}(\xi, t) \exp (x \xi i) d \xi \\
& +\frac{1}{\sqrt{2 \pi}} \int_{-\infty}^{+\infty} \bar{\chi}_{\alpha \gamma}(\xi) \hat{\mathcal{v}}(\xi, t) \exp (x \xi i) d \xi
\end{aligned}
$$

Como $u(x, t)$ depende de $\alpha$ y $\gamma$, vamos a denotarla por $u_{\alpha \gamma}(x, t)$.

\section{B. SOLUCIÓN DE LA ECUACIÓN DEL CALOR, PVI (5)}

Consideramos el PVI

$$
\left\{\begin{array}{l}
\gamma \partial_{t} u-\beta \partial_{x}^{2} u=0 \text { en } \mathbb{R} \times(0,+\infty), \\
u=g_{\gamma} \quad \text { sobre } \mathbb{R} \times\{t=0\},
\end{array}\right.
$$

donde $g_{\gamma}$ es tal que

$$
\hat{g}_{\gamma}(\xi)=\gamma^{2} \tilde{g}(\xi)
$$

Notemos que la elección de los datos iniciales es tal que $\lim _{\alpha \rightarrow 0} \widehat{g}_{\alpha \gamma}(\xi)=\widehat{g}_{\gamma}(\xi)$.

Aplicando transformada con respecto a la variable espacial $x$, obtenemos

$$
\begin{cases}\gamma \partial_{t} \hat{u}+\beta \xi^{2} \hat{u}=0 & \text { parat }>0 \\ \hat{u}=\hat{g}_{\gamma} & \text { para } t=0 .\end{cases}
$$

Tenemos entonces una EDO lineal de primer orden, cuya solución es

$$
\widehat{u}(\xi, t)=C \exp \left(-\frac{\beta \xi^{2}}{\gamma} \mathrm{t}\right)
$$

Usando la condición inicial dada en (25) obtenemos que

$$
\hat{g}_{\gamma}(\xi)=C
$$

luego

$$
\widehat{u}(\xi, t)=\gamma^{2} \tilde{g}(\xi) \exp \left(-\frac{\beta \xi^{2}}{\gamma} t\right)
$$

Aplicando transformada inversa de Fourier, tenemos

$$
\begin{gathered}
u(x, t)=\frac{1}{\sqrt{2 \pi}} \int_{-\infty}^{+\infty} \gamma^{2} \tilde{g}(\xi) \exp (i x \xi \\
\left.-\frac{\beta \xi^{2}}{\gamma} t\right) d \xi
\end{gathered}
$$

Vamos a denotar esta solución por $u_{\gamma}=(x, t)$.

\section{SOLUCIÓN DE LA ECUACIÓN DE ONDA, PVI} (6)

Consideramos el PVI

$$
\left\{\begin{array}{lc}
\alpha \partial_{t}^{2} u-\beta \partial_{x}^{2} u=0 & \text { en } \mathbb{R} \times(0,+\infty) \\
u=g_{\alpha}, \partial_{t} u=h_{\alpha} & \text { sobre } \mathbb{R} \times\{t=0\}
\end{array}\right.
$$

donde $g_{\alpha}(x), h_{\alpha}(x)$ son tales que

$$
\begin{gathered}
\widehat{\boldsymbol{g}}_{\alpha}(\xi)=-4 \alpha \beta \xi^{2} \widetilde{\boldsymbol{g}}(\xi), \\
\widehat{\boldsymbol{h}}_{\alpha}(\xi)=-4 \alpha \beta \xi^{2} \widetilde{\boldsymbol{h}}(\xi) .
\end{gathered}
$$

Vemos que los datos son tales que $\lim _{\gamma \rightarrow 0} \hat{g}_{\alpha \gamma}(\xi)=\hat{g}_{\alpha}(\xi)$, $\lim _{\gamma \rightarrow 0} \hat{h}_{\alpha \gamma}(\xi)=\hat{h}_{\alpha}(\xi)$.

Aplicando transformada de Fourier con respecto a la variable espacial $x$ se obtiene

$$
\begin{cases}\gamma \partial_{t}^{2} \hat{u}+\beta \xi^{2} \hat{u}=0 & \text { para } t>0 \\ \hat{u}=\hat{g}_{\alpha}, \partial_{t} \hat{u}=\hat{h}_{\alpha} & \text { para } t=0\end{cases}
$$


La ecuación característica de la anterior EDO viene dada por

$$
\alpha \lambda^{2}+\beta \xi^{2}=0
$$

y sus raíces son

$$
\begin{aligned}
& \lambda_{1}=i \sqrt{\frac{\beta \xi^{2}}{\alpha}}, \\
& \lambda_{2}=-i \sqrt{\frac{\beta \xi^{2}}{\alpha}} .
\end{aligned}
$$

Entonces la solución de la ecuación es

$$
\begin{aligned}
\widehat{u}(\xi, t)=C_{1} & \exp \left(i \sqrt{\frac{\beta \xi^{2}}{\alpha} t}\right) \\
& +C_{2} \exp \left(-i \sqrt{\frac{\beta \xi^{2}}{\alpha} t}\right) .
\end{aligned}
$$

Considerando las condiciones iniciales tenemos el siguiente sistema

$$
\left\{\begin{array}{c}
\hat{g}_{\alpha}(\xi)=C_{1}+C_{2}, \\
\hat{h}_{\alpha}(\xi)=C_{1} i \sqrt{\frac{\beta \xi^{2}}{\alpha}}-C_{2} i \sqrt{\frac{\beta \xi^{2}}{\alpha}} .
\end{array}\right.
$$

Solucionando este sistema y utilizando (28) y (29) obtenemos

$$
\begin{aligned}
& C_{1}=2 i|\xi| \widetilde{h}(\xi) \sqrt{\alpha^{3} \beta}-2 \alpha \beta \xi^{2} \widetilde{g}(\xi), \\
& C_{2}=-2 i|\xi| \widetilde{h}(\xi) \sqrt{\alpha^{3} \beta}-2 \alpha \beta \xi^{2} \widetilde{g}(\xi)
\end{aligned}
$$

Sustituyendo (31) y (32) en (30) obtenemos

$$
\begin{aligned}
\hat{u}(\xi, t)=\left[2 i|\xi| \sqrt{\alpha^{3} \beta} \tilde{h}(\xi)\right. & \left.-2 \alpha \beta \xi^{2} \tilde{g}(\xi)\right] \exp \left(i \sqrt{\frac{\beta \xi^{2}}{\alpha} t}\right) \\
& -\left[2 i|\xi| \sqrt{\alpha^{3} \beta} \tilde{h}(\xi)\right. \\
& \left.+2 \alpha \beta \xi^{2} \tilde{g}(\xi)\right] \exp \left(-i \sqrt{\frac{\beta \xi^{2}}{\alpha} t}\right) .
\end{aligned}
$$

Aplicando transformada inversa de Fourier nos queda

$$
\begin{aligned}
& u(x, t)=\frac{1}{\sqrt{2 \pi}} \int_{-\infty}^{+\infty}\left[2 i|\xi| \sqrt{\alpha^{3} \beta} \widetilde{h}(\xi)-\right. \\
& \left.2 \alpha \beta \xi^{2} \widetilde{g}(\xi)\right] \exp \left(i \sqrt{\frac{\beta \xi^{2}}{\alpha}} t+i x \xi\right) d \xi- \\
& \frac{1}{\sqrt{2 \pi}} \int_{-\infty}^{+\infty}\left[2 i|\xi| \sqrt{\alpha^{3} \beta} \widetilde{h}(\xi)+\right. \\
& \left.2 \alpha \beta \xi^{2} \widetilde{g}(\xi)\right] \exp \left(i x \xi-i \sqrt{\frac{\beta \xi^{2}}{\alpha}} t\right) d \xi
\end{aligned}
$$

A esta solución vamos a denotarla por $u_{\alpha}(x, t)$

\section{RESULTADO PRINCIPAL}

Nuestro resultado principal es el siguiente.

Teorema 3.1 Sea $u_{\alpha \gamma}$ la solución del PVI (7), dada por la fórmula (23) y para aquellos datos iniciales (8)-(9) con $\Phi_{\alpha \gamma}(\xi)=\gamma^{2}-4 \alpha \beta \xi^{2}$. Entonces se tiene que

i. $\quad$ Si $u_{\gamma}$ es la solución del PVI (24) dada por la fórmula (26), entonces $\lim _{\alpha \rightarrow 0} u_{\alpha \gamma}(x, t)=u_{\gamma}(x, t)$.

ii. Si $u_{\alpha}$ es la solución del PVI (27) dada por la fórmula (33), entonces $\lim _{\gamma \rightarrow 0} u_{\alpha \gamma}(x, t)=u_{\alpha}(x, t)$.

Para demostrar el teorema probemos primero los siguientes lemas:

Lema 3.1 Sean $\hat{U}, \hat{v}, \bar{\chi}_{\alpha \gamma}$ y $\chi_{\alpha \gamma}$ dadas por (16), (20), (21) y (22) respectivamente. Entonces se tiene que

i. $\begin{aligned} & \lim _{\alpha \rightarrow 0} \int_{-\infty}^{+\infty} \chi_{\alpha \gamma}(\xi) \hat{\mathcal{U}}(\xi, t) \cos (x \xi) d \xi= \\ & \int_{-\infty}^{+\infty} \gamma^{2} \tilde{g}(\xi) \exp \left(-\frac{\beta \xi^{2}}{\gamma}\right) \cos (x \xi) d \xi,\end{aligned}$

ii. $\quad \lim _{\alpha \rightarrow 0} \int_{-\infty}^{+\infty} \chi_{\alpha \gamma}(\xi) \hat{\mathcal{U}}(\xi, t) \operatorname{sen}(x \xi) d \xi=$ $\int_{-\infty}^{+\infty} \gamma^{2} \tilde{g}(\xi) \exp \left(-\frac{\beta \xi^{2}}{\gamma}\right) \operatorname{sen}(x \xi) d \xi$,

iii. $\begin{aligned} & \lim _{\alpha \rightarrow 0} \int_{-\infty}^{+\infty} \bar{\chi}_{\alpha \gamma}(\xi) \hat{\mathcal{V}}(\xi, t) \cos (x \xi) d \xi= \\ & \lim _{\alpha \rightarrow 0} \int_{-\infty}^{+\infty} \bar{\chi}_{\alpha \gamma}(\xi) \hat{\mathcal{V}}(\xi, t) \operatorname{sen}(x \xi) d \xi=0 .\end{aligned}$

Demostración.

Demostraremos i-ii. 
Utilizando la desigualdad triangular, la desigualdad media aritmética-geométrica, $\mathrm{y}$ el hecho de que $-\gamma \pm$ $\sqrt{4 \alpha \beta \xi^{2}-\gamma^{2}} \leq 0$ para todo $|\xi| \leq \frac{\gamma}{2 \sqrt{\alpha \beta}}$, se tiene que

$$
\left|\chi_{\alpha \gamma}(\xi) \widehat{u}(\xi, t)\right| \leq M(\xi)
$$

para todo $\xi \in \mathbb{R}$ y $\alpha, \beta, \gamma \in(0, \mathbb{R}]$, donde

$$
\begin{aligned}
M(\xi)=\left(4 R^{3} \xi^{2}\right. & +R^{3} \\
& +R)|\tilde{h}(\xi)|+\left(2 R^{2} \xi^{2}+R^{2}\right. \\
& +1)\left(2 R^{2} \xi^{2}+R^{2}+R+1\right)|\tilde{g}(\xi)| .
\end{aligned}
$$

Dado que $\tilde{g}$ y $\tilde{h}$ son funciones que pertenecen a $S(\mathbb{R})$ tenemos que $\mathrm{M}(\xi)$ es Riemann integrable en $\mathbb{R}$, por tanto integrable en el sentido de Lebesgue en $\mathbb{R}$, ver [1].

De (34) tenemos.

$$
\begin{aligned}
& \left|\chi_{\alpha \gamma}(\xi) \widehat{u}(\xi, t) \cos (x \xi)\right| \leq M(\xi) \\
& \left|\chi_{\alpha \gamma}(\xi) \widehat{u}(\xi, t) \operatorname{sen}(x \xi)\right| \leq M(\xi)
\end{aligned}
$$

para todo $\xi \in \mathbb{R}$ y $\alpha, \beta, \gamma \in(0, \mathbb{R}]$.

Las desigualdades (35) y (36) implican que $\left\{\chi_{\alpha \gamma}(\xi) \hat{u}(\xi, t) \cos (x \xi)\right\}_{\alpha}$ y $\left\{\chi_{\alpha \gamma}(\xi) \hat{u}(\xi, t) \operatorname{sen}(x \xi)\right\}_{\alpha}$ son sucesiones de funciones integrables en el sentido de Lebesgue.

$\mathrm{Al}$ racionalizar $\frac{-\gamma \pm \sqrt{\gamma^{2}-4 \alpha \beta \xi^{2}}}{2 \alpha}$ es fácil ver que

$$
\begin{aligned}
& \lim _{\alpha \rightarrow 0} \chi_{\alpha \gamma}(\xi) \hat{U}(\xi, t) \cos (x \xi) \\
&=\gamma^{2} \tilde{g}(\xi) \exp \left(-\frac{\beta \xi^{2}}{\gamma}\right) \cos (x \xi),
\end{aligned}
$$

$$
\begin{aligned}
\lim _{\alpha \rightarrow 0} \chi_{\alpha \gamma}(\xi) \hat{\mathcal{U}}(\xi, t) \operatorname{sen}(x \xi) & \\
= & \gamma^{2} g(\xi) \exp \left(-\frac{\beta \xi^{2}}{\gamma}\right) \operatorname{sen}(x \xi) .
\end{aligned}
$$

Aplicando el teorema de la convergencia dominada obtenemos

$$
\begin{aligned}
& \lim _{\alpha \rightarrow 0} \int_{-\infty}^{+\infty} \chi_{\alpha \gamma}(\xi) \hat{\mathcal{U}}(\xi, t) \cos (x \xi) d \xi \\
& =\int_{-\infty}^{+\infty} \gamma^{2} \tilde{g}(\xi) \exp \left(-\frac{\beta \xi^{2}}{\gamma}\right) \cos (x \xi) d \xi,
\end{aligned}
$$

$$
\begin{aligned}
& \lim _{\alpha \rightarrow 0} \int_{-\infty}^{+\infty} \chi_{\alpha \gamma}(\xi) \hat{\mathcal{u}}(\xi, t) \operatorname{sen}(x \xi) d \xi \\
& =\int_{-\infty}^{+\infty} \gamma^{2} \tilde{g}(\xi) \exp \left(-\frac{\beta \xi^{2}}{\gamma}\right) \sin (x \xi) d \xi
\end{aligned}
$$

Para la prueba de iii procedemos como en el caso anterior. Observamos entonces que $\bar{\chi}_{\alpha \gamma}(\xi) \hat{v}(\xi, t)$ es una función de valores reales tal que

$$
\left|\bar{\chi}_{\alpha \gamma}(\xi) \widehat{\mathcal{V}}(\xi, t)\right| \leq N(\xi)
$$

para todo $\xi \in \mathbb{R} y \alpha, \beta, \gamma \in(0, \mathbb{R}]$, donde

$$
\begin{aligned}
N(\xi)=\left(6 R^{2} \xi^{2}\right. & +2 R^{2} \\
& +1)|\tilde{g}(\xi)|+\left(R^{3}+2 R^{3} \xi^{2}\right. \\
& +R)|\tilde{h}(\xi)|
\end{aligned}
$$

y $N(\xi)$ resulta una función integrable de Lebesgue.

De (37) tenemos

$$
\begin{aligned}
& \left|\bar{\chi}_{\alpha \gamma}(\xi) \widehat{\mathcal{V}}(\xi, t) \cos (x \xi)\right| \leq N(\xi) \\
& \left|\bar{\chi}_{\alpha \gamma}(\xi) \widehat{\mathcal{V}}(\xi, t) \operatorname{sen}(x \xi)\right| \leq N(\xi)
\end{aligned}
$$

para todo $\xi \in \mathbb{R}$ y $\alpha, \beta, \gamma \in(0, \mathbb{R}]$.

Las desigualdades (38) y (39) implican que $\left\{\bar{\chi}_{\alpha \gamma}(\xi) \hat{\mathcal{V}}(\xi, t) \cos (\chi \xi)\right\}_{\alpha}$ y $\left\{\bar{\chi}_{\alpha \gamma}(\xi) \hat{\mathcal{V}}(\xi, t) \operatorname{sen}(\chi \xi)\right\}_{\alpha} \quad$ son sucesiones de funciones integrables en el sentido de Lebesgue en $\mathbb{R}$.

Por la definición de $\bar{\chi}_{\alpha \gamma}$ en (22) tenemos

$$
\begin{aligned}
& \lim _{\alpha \rightarrow 0} \bar{\chi}_{\alpha \gamma}(\xi) \hat{\mathcal{V}}(\xi, t) \cos (x \xi)=0 \\
& \lim _{\alpha \rightarrow 0} \bar{\chi}_{\alpha \gamma}(\xi) \hat{\mathcal{V}}(\xi, t) \operatorname{sen}(x \xi)=0 .
\end{aligned}
$$

El teorema de la convergencia dominada de Lebesgue nos da

$$
\begin{aligned}
& \lim _{\alpha \rightarrow 0} \int_{-\infty}^{+\infty} \bar{\chi}_{\alpha \gamma}(\xi) \hat{\mathcal{V}}(\xi, t) \cos (x \xi) d \xi=0 \\
& \lim _{\alpha \rightarrow 0} \int_{-\infty}^{+\infty} \bar{\chi}_{\alpha \gamma}(\xi) \hat{\mathcal{V}}(\xi, t) \operatorname{sen}(x \xi) d \xi=0
\end{aligned}
$$

Lema 3.2. Sean $\hat{u}, \hat{v}, \bar{\chi}_{\alpha \gamma}$ y $\chi_{\alpha \gamma}$ dadas por (16), (20), (21) y (22) respectivamente. Entonces se tiene que 

i. $\quad \lim _{\gamma \rightarrow 0} \int_{-\infty}^{+\infty} \bar{\chi}_{\alpha \gamma}(\xi) \hat{\mathcal{V}}(\xi, t) \cos (x \xi) d \xi=$
$\lim _{\gamma \rightarrow 0} \bar{\chi}_{\alpha \gamma}(\xi) \hat{\mathcal{V}}(\xi, t) \operatorname{sen}(x \xi)$
$\int_{-\infty}^{+\infty}\left\{\left[-2 \tilde{g}(\xi) \xi^{2} \alpha \beta+\right.\right.$
$\left.2 i \tilde{h}(\xi)|\xi| \sqrt{\alpha^{3} \beta}\right] \exp \left(\frac{i|\xi| \sqrt{\beta}}{\sqrt{\alpha}} t\right)-$
$\left[2 \widetilde{g}(\xi) \xi^{2} \alpha \beta+\right.$
$\left.\left.2 i \tilde{h}(\xi)|\xi| \sqrt{\alpha^{3} \beta}\right] \exp \left(-\frac{i|\xi| \sqrt{\beta}}{\sqrt{\alpha}} t\right)\right\} \cos (x \xi) d \xi$.
ii. $\quad \lim _{\gamma \rightarrow 0} \int_{-\infty}^{+\infty} \bar{\chi}_{\alpha \gamma}(\xi) \hat{\mathcal{v}}(\xi, t) \operatorname{sen}(x \xi) d \xi=$
$=\left\{\left[-2 \tilde{g}(\xi) \xi^{2} \alpha \beta\right.\right.$
$\left.+2 i \tilde{h}(\xi)|\xi| \sqrt{\alpha^{3} \beta}\right] \exp \left(\frac{i|\xi| \sqrt{\beta}}{\sqrt{\alpha}} t\right)$
$-\left[2 \tilde{g}(\xi) \xi^{2} \alpha \beta\right.$

$$
\left.\left.+2 i \tilde{h}(\xi)|\xi| \sqrt{\alpha^{3} \beta}\right] \exp \left(-\frac{i|\xi| \sqrt{\beta}}{\sqrt{\alpha}} t\right)\right\} \operatorname{sen}(x \xi)
$$

$\int_{-\infty}^{+\infty}\left\{\left[-2 \tilde{g}(\xi) \xi^{2} \alpha \beta+\right.\right.$

$2 i \tilde{h}(\xi)|\xi| \sqrt{\alpha^{3} \beta} \exp \left(\frac{i|\xi| \sqrt{\beta}}{\sqrt{\alpha}} t\right)-$

$\left[2 \tilde{g}(\xi) \xi^{2} \alpha \beta+\right.$

$\left.\left.2 i \tilde{h}(\xi)|\xi| \sqrt{\alpha^{3} \beta}\right] \exp \left(-\frac{i|\xi| \sqrt{\beta}}{\sqrt{\alpha}} t\right)\right\} \operatorname{sen}(x \xi) d \xi$.

Entonces por el teorema de la convergencia dominada de Lebesgue obtenemos

$\lim _{\gamma \rightarrow 0} \int_{-\infty}^{+\infty} \bar{\chi}_{\alpha \gamma}(\xi) \hat{\mathcal{V}}(\xi, t) \cos (x \xi) d \xi$
$=\int_{-\infty}^{+\infty}\left\{\left[-2 \tilde{g}(\xi) \xi^{2} \alpha \beta\right.\right.$
$\left.+2 i \tilde{h}(\xi)|\xi| \sqrt{\alpha^{3} \beta}\right] \exp \left(\frac{i|\xi| \sqrt{\beta}}{\sqrt{\alpha}} t\right)$
$-\left[2 \tilde{g}(\xi) \xi^{2} \alpha \beta\right.$
$\left.\left.+2 i \tilde{h}(\xi)|\xi| \sqrt{\alpha^{3} \beta}\right] \exp \left(-\frac{i|\xi| \sqrt{\beta}}{\sqrt{\alpha}} t\right)\right\} \cos (x \xi) d \xi$

\section{Demostración.}

Demostraremos i-ii).

La desigualdad (37) implica que $\left\{\bar{\chi}_{\alpha \gamma}(\xi) \hat{\mathcal{v}}(\xi, t) \cos (x \xi)\right\}_{\gamma} \mathrm{y}$ $\left\{\bar{\chi}_{\alpha \gamma}(\xi) \hat{v}(\xi, t) \operatorname{sen}(x \xi)\right\}_{\gamma}$ son sucesiones de funciones integrables en el sentido de Lebesgue en $\mathbb{R}$ y puntualmente acotadas por una función no negativa $N(\xi)$, también integrable en el sentido de Lebesgue en $\mathbb{R}$.

Por la definición de $\bar{\chi}_{\alpha \gamma}(\xi)$ en (22) tenemos que

$$
\begin{aligned}
& \lim _{\gamma \rightarrow 0} \bar{\chi}_{\alpha \gamma}(\xi) \hat{\mathcal{V}}(\xi, t) \cos (x \xi) \\
& =\left\{\left[-2 \tilde{g}(\xi) \xi^{2} \alpha \beta\right.\right. \\
& \left.+2 i \tilde{h}(\xi)|\xi| \sqrt{\alpha^{3} \beta}\right] \exp \left(\frac{i|\xi| \sqrt{\beta}}{\sqrt{\alpha}} t\right) \\
& -\left[2 \tilde{g}(\xi) \xi^{2} \alpha \beta\right. \\
& \left.\left.+2 i \tilde{h}(\xi)|\xi| \sqrt{\alpha^{3} \beta}\right] \exp \left(-\frac{i|\xi| \sqrt{\beta}}{\sqrt{\alpha}} t\right)\right\} \cos (x \xi)
\end{aligned}
$$

$\mathrm{y}$

$\lim _{\gamma \rightarrow 0} \int_{-\infty}^{+\infty} \bar{\chi}_{\alpha \gamma}(\xi) \hat{\mathcal{V}}(\xi, t) \operatorname{sen}(x \xi) d \xi$
$=\int_{-\infty}^{+\infty}\left\{\left[-2 \tilde{g}(\xi) \xi^{2} \alpha \beta\right.\right.$
$\left.+2 i \tilde{h}(\xi)|\xi| \sqrt{\alpha^{3} \beta}\right] \exp \left(\frac{i|\xi| \sqrt{\beta}}{\sqrt{\alpha}} t\right)$
$-\left[2 \tilde{g}(\xi) \xi^{2} \alpha \beta\right.$
$\left.\left.+2 i \tilde{h}(\xi)|\xi| \sqrt{\alpha^{3} \beta}\right] \exp \left(-\frac{i|\xi| \sqrt{\beta}}{\sqrt{\alpha}} t\right)\right\} \operatorname{sen}(x \xi) d \xi$

La prueba de iii es similar. En virtud de (34) $\left\{\chi_{\alpha \gamma}(\xi) \hat{u}(\xi, t) \cos (x \xi)\right\}_{\gamma},\left\{\chi_{\alpha \gamma}(\xi) \hat{u}(\xi, t) \sin (x \xi)\right\}_{\gamma}$ у $M(\xi)$ son una clase de funciones integrables en el sentido de Lebesgue.

Ahora la definición de $\chi_{\alpha \gamma}(\xi)$ en (21) nos lleva a que

$$
\lim _{\alpha \rightarrow 0} \chi_{\alpha \gamma}(\xi) \widehat{u}(\xi, t) \cos (x \xi)=0,
$$




$$
\lim _{\alpha \rightarrow 0} \chi_{\alpha \gamma}(\xi) \widehat{u}(\xi, t) \sin (x \xi)=0 .
$$

Una vez más, el teorema de la convergencia dominada nos da el resultado

$$
\begin{aligned}
& \lim _{\gamma \rightarrow 0} \int_{-\infty}^{+\infty} \chi_{\alpha \gamma}(\xi) \hat{u}(\xi, t) \cos (x \xi) d \xi=0, \\
& \lim _{\gamma \rightarrow 0} \int_{-\infty}^{+\infty} \chi_{\alpha \gamma}(\xi) \hat{\mathcal{u}}(\xi, t) \operatorname{sen}(x \xi) d \xi=0 .
\end{aligned}
$$

\section{Demostración del teorema 3.1:}

i) Por el lema 3.1 (i)-(ii) tenemos que

$$
\begin{aligned}
& \lim _{\alpha \rightarrow 0} \frac{1}{\sqrt{2 \pi}} \int_{-\infty}^{+\infty} \chi_{\alpha \gamma}(\xi) \widehat{u}(\xi, t) \exp (x \xi i) d \xi \\
& =\frac{1}{\sqrt{2 \pi}} \int_{-\infty}^{+\infty} \gamma^{2} \widetilde{g}(\xi) \exp \left(i x \xi-\frac{\beta \xi^{2}}{\gamma}\right) d \xi .
\end{aligned}
$$

Del lema 3.1(iii) tenemos

$$
\begin{gathered}
\lim _{\alpha \rightarrow 0} \frac{1}{\sqrt{2 \pi}} \int_{-\infty}^{+\infty} \bar{\chi}_{\alpha \gamma}(\xi) \widehat{\mathcal{V}}(\xi, t) \exp (x \xi i) d \xi \\
=0 .
\end{gathered}
$$

Por tanto, (23), (42) y (43) implican

$$
\begin{aligned}
\lim _{\alpha \rightarrow 0} u_{\alpha \gamma}(x, t) & =\frac{1}{\sqrt{2 \pi}} \int_{-\infty}^{+\infty} \gamma^{2} \tilde{g}(\xi) \exp (i x \xi \\
& \left.-\frac{\beta \xi^{2}}{\gamma}\right) d \xi=u_{\gamma}(x, t) .
\end{aligned}
$$

ii) Como en (i), el lema 3.2 nos da

$$
\begin{aligned}
& \lim _{\gamma \rightarrow 0} \frac{1}{\sqrt{2 \pi}} \int_{-\infty}^{+\infty} \bar{\chi}_{\alpha \gamma}(\xi) \widehat{\mathcal{v}}(\xi, t) \exp (x \xi i) d \xi \\
& =\frac{1}{\sqrt{2 \pi}} \int_{-\infty}^{+\infty}\left[-2 \widetilde{g}(\xi) \xi^{2} \alpha \beta\right. \\
& \left.+2 i \widetilde{h}(\xi)|\xi| \sqrt{\alpha^{3} \beta}\right] \exp \left(\frac{i|\xi| \sqrt{\beta}}{\sqrt{\alpha}}+i x \xi\right) d \xi \\
& -\frac{1}{\sqrt{2 \pi}} \int_{-\infty}^{+\infty}\left[2 \widetilde{g}(\xi) \xi^{2} \alpha \beta\right. \\
& \left.+2 i \widetilde{h}(\xi)|\xi| \sqrt{\alpha^{3} \beta}\right] \exp \left(-\frac{i|\xi| \sqrt{\beta}}{\sqrt{\alpha}}\right. \\
& +i x \xi) d \xi,
\end{aligned}
$$

$$
\begin{gathered}
\lim _{\gamma \rightarrow 0} \frac{1}{\sqrt{2 \pi}} \int_{-\infty}^{+\infty} X_{\alpha \gamma}(\xi) \widehat{u}(\xi, t) \exp (x \xi i) d \xi \\
=0 .
\end{gathered}
$$

De (23), (44) y (45) se sigue el resultado

$$
\lim _{\gamma \rightarrow 0} u_{\alpha \gamma}(x, t)=u_{\alpha}(x, t) .
$$

Corolario 3.1. Para $u_{\alpha \gamma}, u_{\alpha}, u_{\gamma}$ como en el teorema 3.1, tenemos que

$$
\lim _{(\alpha, \gamma) \rightarrow(0,0)}\left|u_{\alpha}(x, t)-u_{\gamma}(x, t)\right|=0 .
$$

Demostración. Por el teorema 3.1 tenemos los siguientes límites:

$$
\begin{aligned}
& \lim _{\alpha \rightarrow 0} u_{\alpha \gamma}(x, t)=u_{\gamma}(x, t), \\
& \lim _{\gamma \rightarrow 0} u_{\alpha \gamma}(x, t)=u_{\alpha}(x, t) .
\end{aligned}
$$

Entonces, dado cualquier $\varepsilon>0$ tenemos que

$$
\begin{aligned}
& \left|u_{\gamma}(x, t)-u_{\alpha \gamma}(x, t)\right|<\frac{\varepsilon}{2}, \\
& \left|u_{\alpha}(x, t)-u_{\alpha \gamma}(x, t)\right|<\frac{\varepsilon}{2}
\end{aligned}
$$

para $\alpha$ y $\gamma$ en $(0, R]$ suficientemente pequeños.

Por tanto

$$
\begin{aligned}
\mid u_{\gamma}(x, t)-u_{\alpha} & (x, t) \mid \\
& \leq\left|u_{\gamma}(x, t)-u_{\alpha \gamma}(x, t)\right| \\
& +\left|u_{\alpha}(x, t)-u_{\alpha \gamma}(x, t)\right| \\
& <\frac{\varepsilon}{2}+\frac{\varepsilon}{2} \\
& =\varepsilon
\end{aligned}
$$

para $\alpha$ y $\gamma$ en $(0, R]$ suficientemente pequeños.

\section{CONCLUSIONES}

Hemos probado convergencia puntual de las soluciones $u_{\alpha \gamma}$ del PVI (7) para soluciones $u_{\gamma}$ y $u_{\alpha}$ del PVI (24) y (27) respectivamente, siempre y cuando los datos iniciales asociados a la ecuación del calor (24) y a la ecuación de onda (27) sean los límites de baja densidad y límite no viscoso respectivamente, de los datos iniciales de la ecuación del telégrafo (7). 
Esta primera aproximación dependió fuertemente del símbolo $\Phi_{\alpha \gamma}(\xi)$, el discriminante de la ecuación característica asociada a la EDO derivada de la EDP (7) vía la Transformada de Fourier.

Creemos que el resultado puede mejorarse en el sentido de que los datos iniciales no necesitan estar en la clase $S(\mathbb{R})$ moduladas por el símbolo $\Phi$ y que la convergencia pueda obtenerse en el espacio de funciones continuas, es decir, la convergencia puede ser uniforme.

\section{AGRADECIMIENTOS}

Este trabajo fue apoyado por la Vicerrectoría de Investigación y la Dirección de Investigaciones de la Universidad Nacional de Colombia Sede Manizales, DIMA en el marco del Semillero de Investigación en Ecuaciones Diferenciales con código HERMES 13496

\section{REFERENCIAS}

[1] Tom M. Apostol, Mathematical Analysis, second edition, Reverté, 2009.

[2] L. Evans, Partial Differential Equations, American Mathematical Society, 1998.

[3] V. De Magalhaes Iório, EDP: Um Curso de Graduação, Terceira Edicão, Coleçao Matemática Universitaria IMPA, 2012.

[4] R. Iório, V. De Magalhaes Iório, Fourier Analysis and Partial Differential Equations, Cambridge University Press, 2001.

[5] J. Lopez Gondar, R. Cipolatti. Iniciação á Física Matematica, Coleção Matematica e Aplicações IMPA, 2011.

[6] I. Peral Alonso. Primer Curso de Ecuaciones en Derivadas Parciales, Universidad Autónoma de Madrid, 2004.

[7] W. Rudin. Principles of mathematical analysis, McGrawHill, 1980. 